\title{
Bilateral Tele-Rehabilitation System with Electrical Stimulation by Using Cloud Service
}

\author{
Yasunori Kawai ${ }^{1+}$, Koudai Houga ${ }^{1}$, Hiroyuki Kawai ${ }^{2}$, and Takanori Miyoshi ${ }^{3}$ \\ ${ }^{1}$ Department of Electrical Engineering, National Institute of Technology, Ishikawa College, Japan \\ ${ }^{2}$ Department of Robotics, Kanazawa Institute of Technology, Japan \\ ${ }^{3}$ Department of System Safety, Nagaoka University of Technology, Japan
}

\begin{abstract}
This paper considers a bilateral tele-rehabilitation system with electrical stimulation for a human lower limb by using the Amazon Web Services Internet of Things (AWS IoT) as a cloud service. The scattering matrix method can guarantee the stability for the time delay in the bilateral tele-rehabilitation. This paper applies the AWS IoT to the bilateral tele-rehabilitation system. Because the cross certification and the cipher are provided in AWS IoT, the cybersecurity is considered compared to the HTTP method in the previous research. The experimental results show that the stability can be compensated even if the time delay exist. However, the error between the paddle angle and the knee angle exist.
\end{abstract}

Keywords: Bilateral Control, Tele-Rehabilitation, Cloud Service

\section{Introduction}

The electrical stimulation is known as Functional Electrical Stimulation (FES) to improve the motor function of a human. FES makes muscle contraction by external electrical impulses in the same way as electrical impulses from the brain. When the stimulation is controlled, a desired movement can be achieved [1].

The tele-rehabilitation with the electrical stimulation has been proposed in [2]. The tele-rehabilitation indicates that a physical therapist rehabilitates a patient from the remote location. In [2], the therapist uses the haptic device, the lower limb of the patient is controlled by the electrical stimulation. The contact force and position information are communicated between the patient and the therapist. The lower limb of the patient and haptic device are controlled bilaterally. It is called as the bilateral tele-rehabilitation system.

In the previous research, the tele-rehabilitation system with motor-assisted device has been developed in [3]. The physical therapist rehabilitates the patient by the motor-assisted device while the therapist watches the patient through the monitor. It is difficult to feel the condition of the patient. As the another bilateral teleoperation, the tele-rehabilitation system with the haptic device has been developed in [4]. The therapist rehabilitates the patient by grasping the rehabilitation device and the haptic device, respectively. The force and velocity information are communicated between the patient and the therapist. However, the velocity information is not better than the position information in [2]

The stabilization methods for the time delay have been investigated in the bilateral teleoperation, because the bilateral teleoperation is the closed loop system. The passivity-based approach is proposed in [5]-[7] by using the scattering transformation and the wave variable. The stability is guaranteed by the passivity of the operator, the tele-operator, and the communication block including time delays. As one of the non-passive approach for the time delay, the scattering matrix method has been proposed in [2],[8]. The stability is guaranteed based on the norm of the operator and the tele-operator.

+ Corresponding author. Tel.: +81-76-288-8110

E-mail address: y_kawai@ishikawa-nct.ac.jp 
This paper considers the experimental verification of the bilateral tele-rehabilitation system using Amazon Web Services Internet of Things (AWS IoT). The main contribution is to apply the AWS IoT to the bilateral tele-rehabilitation system. Because the cross certification and the cipher are provided in AWS IoT, the cybersecurity is considered compared to the HTTP method in the previous research in [9]. The stability is confirmed when the time delay exists by the AWS IoT in the experiment.

\section{Bilateral Tele-rehabilitation System Using Scattering Matrix Method}

This section reviews the bilateral tele-rehabilitation system based on the scattering matrix method in the previous work of [2]. Fig. 1 shows the bilateral tele-rehabilitation system of the human lower limb. A force $f_{h}(t) \in R$ is provided to the paddle by the therapist. The force information is sent to a patient through the Internet. In the patient side, the electrical stimulus signal is made according to the received force information. The stimulus signal is provided to the quadriceps femoris muscle group $e_{2}$ and $e_{3}$. The muscle contraction induced by the electrical stimulation yields the leg extension. At the same time, the information of the patient's knee angle $q_{s}(t) \in R$ is sent to the therapist through the Internet. In the therapist side, the paddle's angle $q_{m}(t) \in R$ is controlled to be the same angle as the patient's knee angle $q_{s}(t)$ by the motor. Then, the therapist can feel the motion of the patient's lower limb by the reaction force from the paddle.

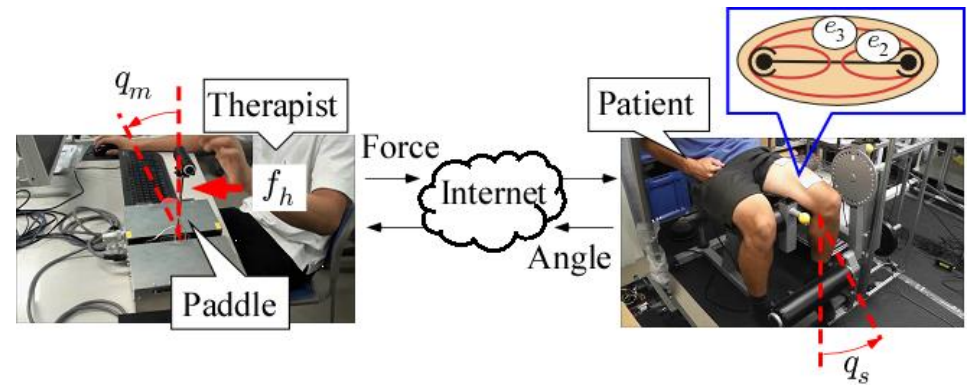

Fig. 1: Bilateral tele-rehabilitation system of the human lower limb.

The bilateral tele-rehabilitation system using the scattering matrix method is illustrated in Fig. 2. The therapist model is $G_{m m}(s)$, the patient model is $G_{s s}(s)$, the wave filters are $W_{m}(s)$ and $W_{s}(s)$, time delays are $T_{1}(t)$ and $T_{2}(t)$ in the communication by the Internet. The force $f_{h}(t)$ is indicated in Fig. 1, the disturbance is $f_{e}(t) \in R$.

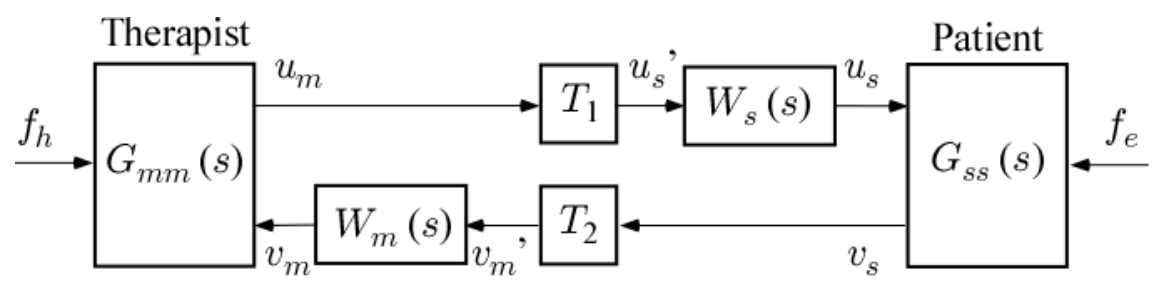

Fig. 2: Bilateral tele-rehabilitation system using the scattering matrix method.

The therapist model $G_{m m}(s)$ in Fig. 2 is shown in Fig. 3. The paddle model is $P_{m}(s)$, the friction force model of the paddle is $P_{f}(s)$, the feedback controller is $K_{m}(s)$, the phase-lag filter is $G_{c m}(s)$, the square box which is drawn by the dashed line is the scattering transformation, where $b \in R$ is the design parameter. The paddle angle is $q_{m}(t)$, the reference angle of the paddle angle is $q_{r m}(t) \in R$ which is equal to the past patient's knee angle $q_{s}\left(t-T_{2}\right)$, the force which is measured by the force sensor on the paddle is $f_{m}(t) \in R$.

The patient model $G_{s s}(s)$ in Fig. 2 is shown in Fig. 4. The patient's lower limb model is $P_{s}(s)$, the phaselead filter is $G_{c s}(s)$, the square box written by the dashed line is the scattering transformation. The phase-lead filter $G_{c s}(s)$ is needed to stabilize the patient, because the feedback controller doesn't exist in the patient side. The patient's knee angle is $q_{s}(t)$, the force $f_{s}(t) \in R$ is equal to the force $f_{m}\left(t-T_{1}\right)$. The force $f_{s}(t)$ is provided by the muscles $e_{2}$ and $e_{3}$ by the electrical stimulation.

The stability condition of the bilateral teleoperation system based on the scattering matrix method has already been proposed in [8] as follows by using the small gain theorem

$$
\left\|G_{m m}(s) W_{m}(s)\right\|_{\infty} \cdot\left\|G_{s s}(s) W_{s}(s)\right\|_{\infty} \leq 1 .
$$


The stability condition Eq. (1) means that the stability condition can be evaluated by using $H_{\infty}$ norm regardless of the length of the time delay. The control objectives are indicated as follows;

(1) The paddle's angle $q_{m}(t)$ is the same angle as the patient's knee angle $q_{s}(t)$.

(2) The therapist feels the reaction force from the paddle.

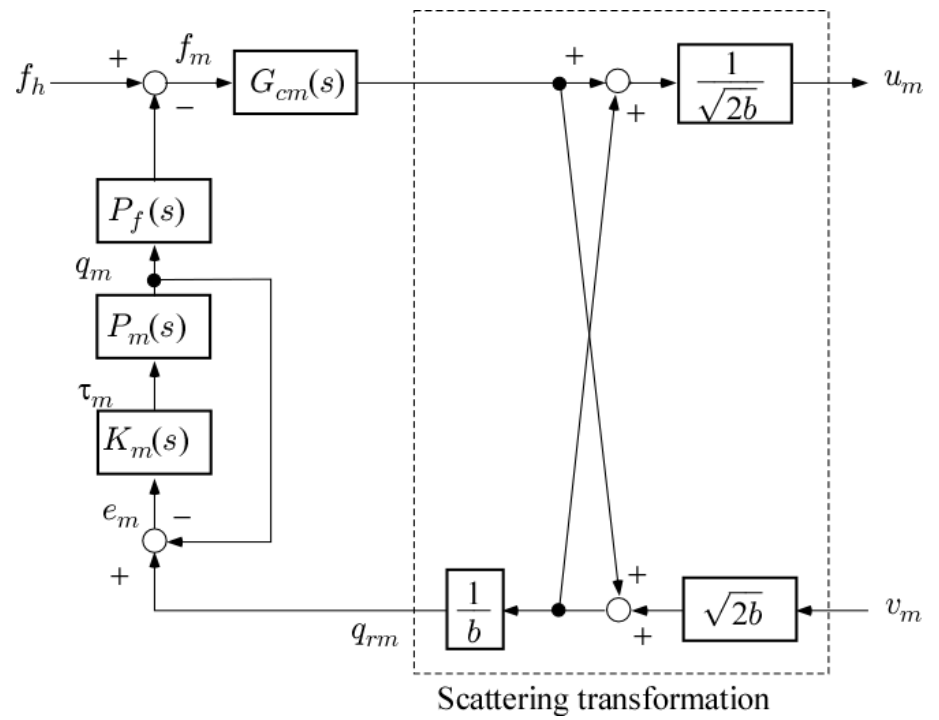

Fig. 3: Therapist model $G_{m m}(s)$ in Fig. 2.

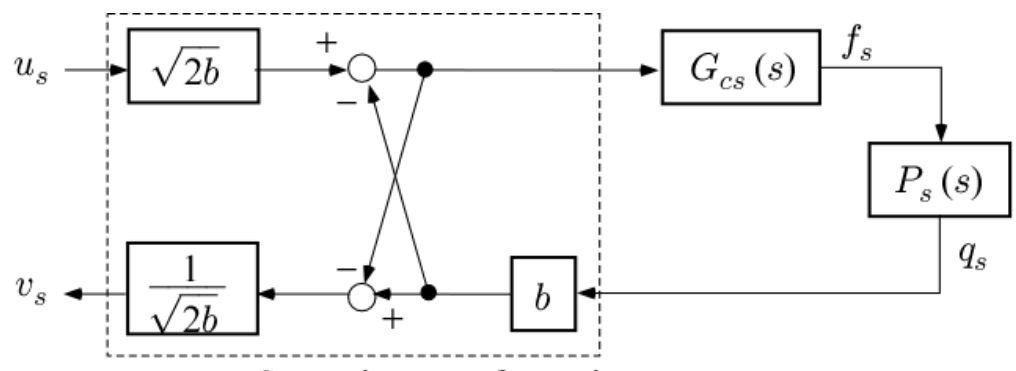

Scattering transformation

Fig. 4: Patient model $G_{s s}(s)$ in Fig. 2.

\section{Experimental Setup}

The experimental equipment of the tele-rehabilitation system is shown in Fig. 5. In the therapist side, the contact force $f_{h}(t)$ is measured by using the force sensor where the force sensor value is $f_{m}(t)$. The paddle angle $q_{m}(t)$ and reaction force $\tau_{m}(t)$ are obtained by utilizing the encoder and DC motor, respectively. In the patient side, the patient sits on the leg extension machine with a weight 5 [LBS] $(=2.3[\mathrm{~kg}])$. The knee angle $q_{s}(t)$ is measured by using the encoder. The contact force $f_{s}(t)$ is converted to the electrical stimulus signal $\tau_{s}(t)$ by utilizing the RehaStim (HASOMED GmbH). The PC with the signal processing board Q8-USB (Quanser) is connected to the therapist side and patient side. The communication between the PC and the AWS IoT are implemented by the "Publish" and "Subscribe". In the AWS IoT, the things Topic1 and Topic2 are made. The information $f_{m}(t)$ and $q_{s}(t)$ are sent to the Topic 1 and Topic2 by "Publish". When the "Publish" is implemented, the PC takes the information $f_{s}(t)$ and $q_{s}(t)$ by "Subscribe". The design parameters are designed as follows;

$$
\begin{gathered}
G_{c m}(s)=\frac{0.1 s+5}{s+5}, G_{c s}(s)=G_{c m}^{-1}(s)=\frac{s+5}{0.1 s+5}, \\
K_{m}(s)=2+\frac{2}{s}+0.1 s, \\
W_{m}(s)=W_{s}(s)=\frac{1}{(0.05 s+1)}, \quad b=20 .
\end{gathered}
$$




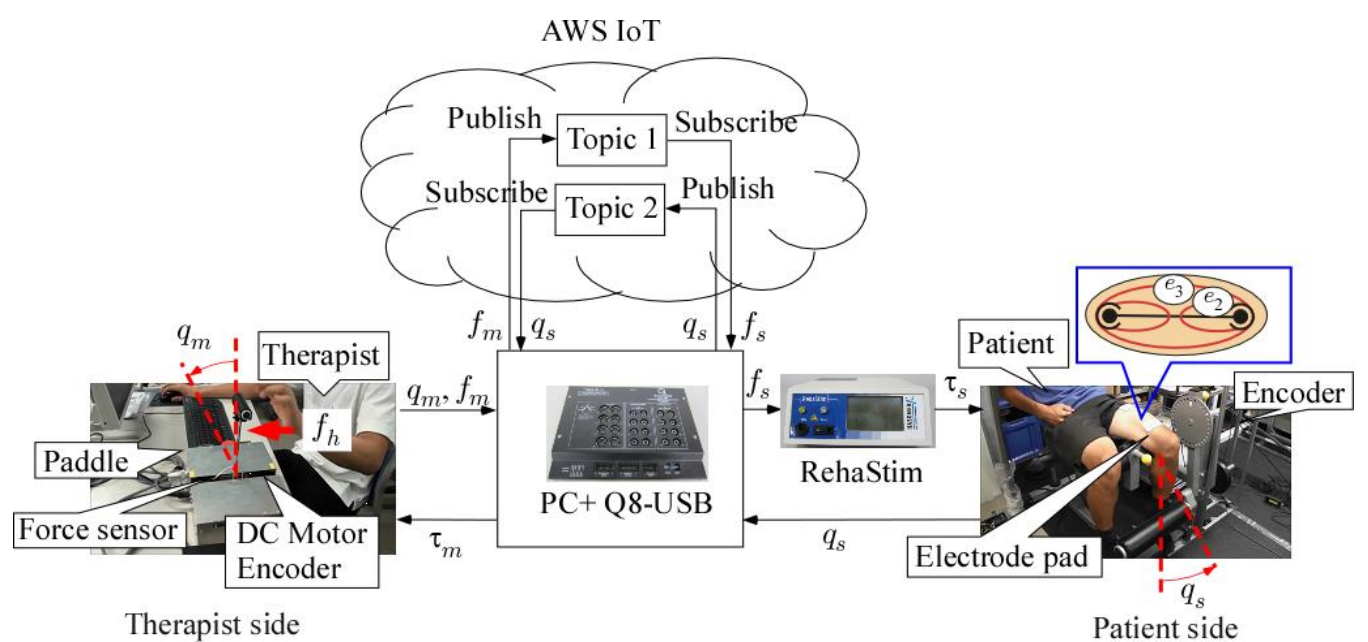

Fig. 5: Experimental equipment.

\section{Experimental Result}

The experimental results are shown in Figs. 6-10. The therapist provides the contact force $f_{h}(t)$ to the paddle. The sensor value $f_{m}(t)$ is indicated in Fig. 6 , where the dashed line is $f_{m}(t)$. The solid line is $f_{s}(t)$ which is same as $f_{m}\left(t-T_{1}(t)\right)$ in Fig. 6. The forces $f_{s}(t)$ and $f_{m}(t)$ almost have the same value. The magnitude of the electrical stimulation $\tau_{s}(t)$ according to the force $f_{s}(t)$ is illustrated in Fig. 7. Then, the knee angle of the patient $q_{s}(t)$ is illustrated in Fig. 8, where the solid line is $q_{s}(t)$. The dashed line in Fig. 8 is the paddle angle $q_{m}(t)$. The reaction force $\tau_{m}(t)$ is shown in Fig. 9. The time delays are indicated in Fig. 10. The time delays $T_{1}(t)$ and $T_{2}(t)$ are the required time from the therapist to the patient and the patient to the therapist, respectively. The average time delay is 0.2 [s]. Though the error between the paddle angle $q_{m}(t)$ and the knee angle $q_{s}(t)$ exists for $3 \leq t \leq 5$, the stability is compensated even if the time delay exist.

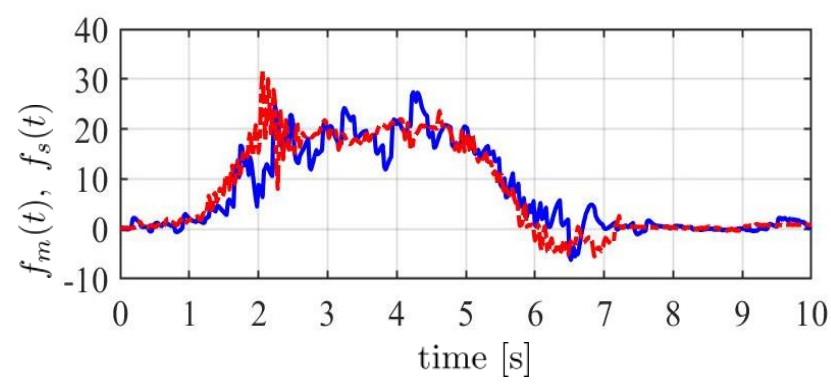

Fig. 6: Time response of the force $f_{m}(t)$ and the force $f_{s}(t)$. (solid: $f_{s}(t)$, dashed: $f_{m}(t)$ )

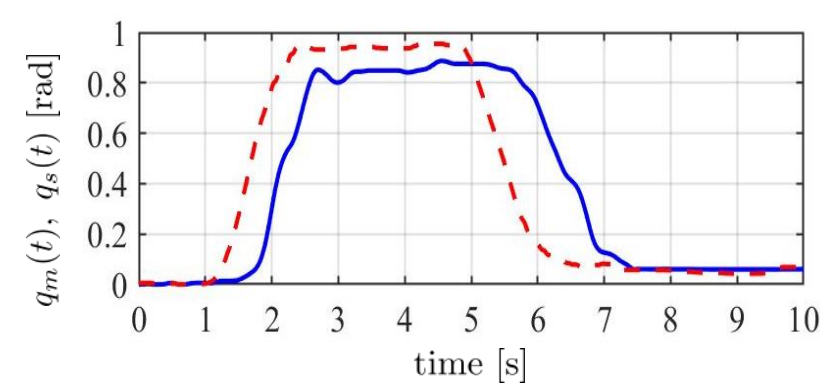

Fig. 8: Time response of the paddle angle $q_{m}(t)$ and the knee angle $q_{s}(t)$. (solid: $q_{s}(t)$, dashed: $q_{m}(t)$ )

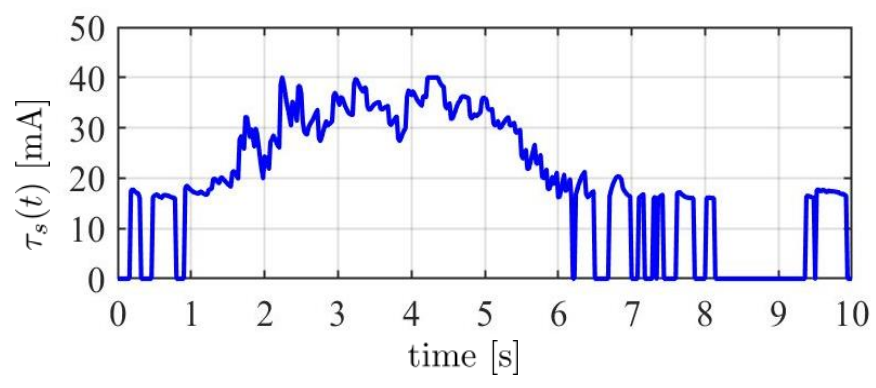

Fig. 7: Time response of the magnitude of the electrical stimulation $\tau_{s}(t)$

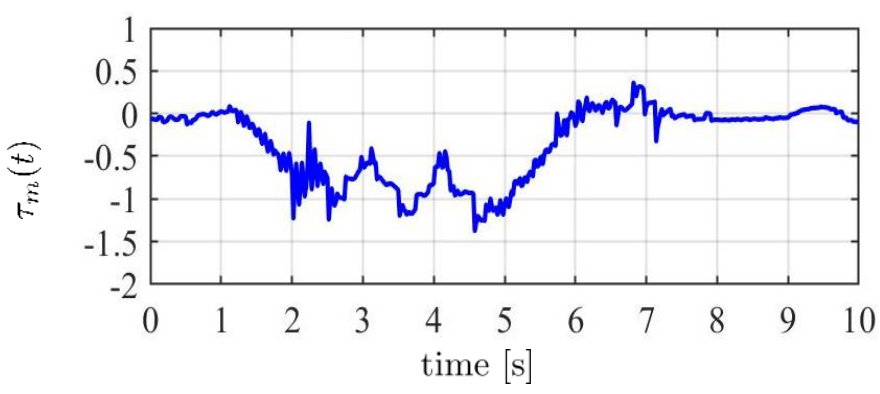

Fig. 9: Time response of the reaction force $\tau_{m}(t)$. 


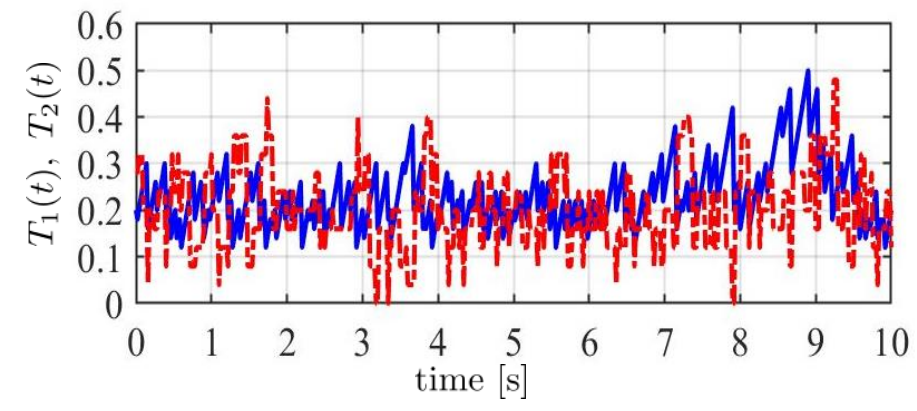

Fig. 10: Time response of the time delays $T_{1}(t), T_{2}(t)$. (solid: $T_{1}(t)$, dashed: $T_{2}(t)$ )

\section{Conclusion}

This paper considered the experimental verification of the bilateral tele-rehabilitation system using AWS IoT. The therapist side and the patient side can be communicated through two things on AWS IoT. The error between the paddle angle and the knee angle exist. However, the stability can be compensated for the average time delay $0.2[\mathrm{~s}]$.

\section{References}

[1] C. T. Freeman, E. Rogers, A. Hughes, J. H. Burridge, and K. L. Meadmore, "Iterative Learning Control in Health Care: Electrical Stimulation and Robotic-Assisted Upper-Limb Stroke Rehabilitation," IEEE Control Systems Magazine, Vol. 32, No. 1, pp. 18-43, 2012.

[2] Y. Kawai, K. Honda, H. Kawai, T. Miyoshi, and M. Fujita, "Tele-Rehabilitation System for Human Lower Limb using Electrical Stimulation based on Bilateral Teleoperation," in Proc. the 2017 IEEE Conference on Control Technology and Applications (CCTA), pp. 1446-1451, 2017.

[3] J. Bae, W. Zhang, and M. Tomizuka, "Network-Based Rehabilitation System for Improved Mobility and TeleRehabilitation," IEEE Trans. on Control Systems Technology, Vol. 21, No. 5, pp. 1980-1987, 2013.

[4] S. F. Atashzar and M. Shahbazi and M. Tavakoli, and R. V. Patel, "A Passivity-Based Approach for Stable Patient-Robot Interaction in Haptics-Enabled Rehabilitation Systems: Modulated Time-Domain Passivity Control," IEEE Trans. on Control Systems Technology, Vol. 25, No. 3, pp. 991-1006, 2017.

[5] T. Hatanaka and N. Chopra and M. Fujita, and M. W. Spong, Passivity Based Control and Estimation in Networked Robotics, Springer International Publishing, Communications and Control Engineering Series, 2015.

[6] P. F. Hokayem and M. W. Spong, "Bilateral Teleoperation: An Historical Survey," Automatica, Vol. 42, No. 12, pp. 2035-2057, 2006.

[7] E. Nuno and L. Basanez, and R. Ortega, "Passivity-based control for bilateral teleoperation: A tutorial," Automatica, Vol. 47, No. 3, pp. 485-495, 2011.

[8] T. Miyoshi, K. Terashima, and M. Buss, "A Design Method of Wave Filter for Stabilizing Non-Passive Operating System," in Proc. the 2006 IEEE International Conference on Control Applications, pp. 1318-1324, 2006.

[9] Y. Kawai, K. Honda, and M. Koshino, "Bilateral Tele-Rehabilitation System with Electrical Stimulation through Cloud Server," in Proc. the 2019 IEEE International Conference on Consumer Electronics-Taiwan (ICCE-TW), 2019 . 